\title{
PECVD Silicon Nitride Passivation on Boron Emitter: The Analysis of Electrostatic Charge on the Interface Properties
}

\author{
Natalita M. Nursam, Yongling Ren, and Klaus J. Weber \\ Centre for Sustainable Energy System, School of Engineering, The Australian National University, Building 32 North Road, \\ Acton, Canberra 0200, Australia \\ Correspondence should be addressed to Natalita M. Nursam, natalita.nursam@anu.edu.au
}

Received 9 March 2010; Accepted 10 June 2010

Academic Editor: Chang Sun

Copyright ( $\odot 2010$ Natalita M. Nursam et al. This is an open access article distributed under the Creative Commons Attribution License, which permits unrestricted use, distribution, and reproduction in any medium, provided the original work is properly cited.

The dependence of surface recombination of boron diffused and undiffused silicon surfaces passivated with a-SiN $: \mathrm{H}$ on the net charge density is investigated in detail. The films are deposited by plasma-enhanced chemical vapour deposition using a $2.45 \mathrm{GHz}$ microwave remote plasma system. The surface charge density on the samples is varied by depositing charge using a corona discharge chamber. Excess carrier lifetime, capacitance-voltage, and Kelvin probe measurements are combined to determine the surface recombination velocity and emitter saturation current density as a function of net charge density. Our results show that the application of negative charge causes a substantial reduction in the surface recombination of samples with boron diffused emitters, even for high boron surface concentrations of $5 \times 10^{19} \mathrm{~cm}^{-3}$. The significant difference observed in surface recombination between boron diffused and undiffused sample under accumulation implies that the presence of boron diffusion has results in some degradation of the $\mathrm{Si}_{-} \mathrm{SiN}_{x}$ interface. Further, (111) oriented surfaces appear more sensitive to the boron surface concentration than (100) oriented surfaces.

\section{Introduction}

The incorporation of silicon nitride $\left(\mathrm{SiN}_{x}\right)$ films into semiconductor devices, and in particularly solar cells, has become an increasingly important topic. This is partly due to their favorable characteristics, which allow them to act as antireflection coatings as well as passivation layers. For the case of PECVD deposited nitride, the film properties can be varied over a broad range, and the films contain a high concentration of hydrogen atoms that can be used-topassivate bulk defects. Given the growing interest in solar cells based on n-type silicon substrates [1], the passivation of p-type emitters has become more important and, therefore, the application of PECVD $\mathrm{SiN}_{x}$ films on this type of emitter has emerged as a topic of interest as well.

PECVD $\mathrm{SiN}_{x}$ generally contains a moderate to high density of positive fixed charge located at or near the Si$\mathrm{SiN}_{x}$ interface. This positive charge is beneficial for the passivation of lowly doped or $\mathrm{n}$ doped surfaces but is generally undesirable on heavily doped $\mathrm{p}$ type $\left(p^{+}\right)$surfaces, such as boron diffused emitters, as the positive charge results in a reduction in hole concentration at the Si surface and consequently an increase in surface recombination velocity. While recent studies have demonstrated that PECVD nitride could actually provide excellent passivation of $\mathrm{B}$ diffused emitter structures when an appropriate postannealing process was carried out [2], the paper did not provide details of the charge density in the $\mathrm{SiN}_{x}$ film, or the B surface concentration. Hence, the reason for the excellent reported surface passivation has not been clearly established. Moreover, the exact dependence of the surface recombination rate on the combination of charge density in the PECVD nitride film and the $\mathrm{p}^{+}$diffused emitter profile has not been explored in detail. Neither does much information exist on whether the presence of a B diffusion results in significant changes to the $\mathrm{Si}_{-} \mathrm{SiN}_{x}$ interface region (such as the creation of more interface defects). This paper aims to investigate these issues. 


\section{Impact of Charge on Surface Recombination}

The surface recombination velocity $(S)$ is defined by the following expression:

$$
\begin{gathered}
U_{s} \equiv S\left(\Delta n_{s}\right) \cdot \Delta n_{s}, \\
S\left(\Delta n_{S}\right)=v_{t h} \int_{E_{v}}^{E_{c}} \frac{D_{i t}\left(E_{t}\right)\left(n_{0}+p_{0}+\Delta n_{s}\right)}{1 /\left(\sigma_{p}\left(E_{t}\right)\right)\left(n_{S}+n_{i} \exp \left(\left(E_{t}-E_{i}\right) / k T\right)\right)+1 /\left(\sigma_{n}\left(E_{t}\right)\right)\left(p_{S}+n_{i} \exp -\left(\left(E_{t}-E_{i}\right) / k T\right)\right)} d E_{t},
\end{gathered}
$$

where $U_{s}$ is the recombination rate at the surface and $\Delta n_{s}$ is the excess surface carrier concentration. Using the ShockleyRead-Hall (SRH) equation, $S$ can be expressed as [3] where $v_{\text {th }}$ is the thermal velocity, $n_{0}$ and $p_{0}$ are the electron and hole concentration under equilibrium, $n_{i}$ is the intrinsic carrier concentration, $n_{s}$ and $p_{s}$ are the electron and hole concentrations at the surface, $E_{t}$ is the interface defects energy level, $D_{i t}\left(E_{t}\right)$ is the energy-dependent interface defects density, and $\sigma_{n}\left(E_{t}\right)$ and $\sigma_{p}\left(E_{t}\right)$ are the energy-dependent for electrons and holes, respectively. Examination of (2) shows that, while $S$ is in general a function of $\Delta n_{s}, S$ can be considered to be independent of $\Delta n_{s}$ under low level injection conditions at the surface. Furthermore, for high surface majority carrier concentrations $\left(>10^{18} \mathrm{~cm}^{-3}\right), S$ is expected to be only weakly dependent on $p_{s}$ if the interface properties do not change with $p_{s}$.

Surface recombination can be minimized by reducing the concentration of one type of carrier at the surface, either electrons or holes. This can be achieved by creating either accumulation or strong inversion conditions at the surface. Further examination of the SRH equation shows that, for defects with energy levels that are not close to the band edges and with a capture cross section ratio $R=\sigma_{n} / \sigma_{p}$, the peak in recombination will occur when the carrier concentration at the surface is $p_{S} / n_{S}=R$, where $p_{S}$ and $n_{S}$ are the surface hole and electron concentrations, respectively [4]. For approximately equal capture cross sections, the peak will occur when $p_{S} \approx n_{S}$, that is, when the surface is in depletion. Surface recombination as a function of charge density can be modelled using the extended SRH formalism developed by Girisch et al. [5] and Aberle et al. [6]. An example of this model is given in Figure 1, showing the effect of surface charge upon surface recombination rate for samples with different surface doping concentrations $\left(N_{s}\right)$. This model predicts that, under strong inversion or accumulation, surface recombination will become independent of the surface doping, and relatively weakly dependent on charge density. This suggests that a comparison of samples under deep inversion or accumulation conditions can be used to directly compare the interface quality (in terms of interface defect density distribution and capture cross sections) of different samples.

Investigations of surface recombination as a function of surface charge density can be performed by applying a gate voltage on a suitable metal-insulator-semiconductor (MIS) structure, or by depositing corona charges on the surfaces of samples. Several such investigations have been carried out for the $\mathrm{Si}_{-} \mathrm{SiO}_{2}$ interface [7-10], where generally good qualitative agreement between experimental and modelled results has been obtained. Few publications report on similar studies on the Si-PECVD $\operatorname{SiN}_{x}$ system, but the results are similar to those obtained on $\mathrm{Si}_{-} \mathrm{SiO}_{2}$ [11]. An important and consistently observed feature in all the experimental results is the saturation (rather than a continued reduction, as predicted by models) of surface recombination for sufficiently large densities of positive and negative charge. For $\mathrm{Si}_{-} \mathrm{SiO}_{2}$, saturation occurs at a net charge density of less than $5 \times 10^{12} \mathrm{~cm}^{-2}$, or a majority carrier concentration of $5 \times 10^{19} \mathrm{~cm}^{-3}[4,10,12]$. The majority carrier concentration here means the concentration of the majority carrier taking into account the effect of the application of surface charge. For example, for a B diffused surface with a very high positive charge density, the majority carriers would be electrons.

\section{Experimental}

Samples used for lifetime measurements were Float Zoned (FZ), n-type, (100), 40-60 ohm.cm, 500 um thick $c$-Si wafers. These samples were etched (removing at least $15 \mu \mathrm{m}$ per side) in $\mathrm{HNO}_{3}$ and $\mathrm{HF}$ solution to remove any saw damage. After receiving a standard RCA clean, selected samples were subjected to a $\mathrm{B}$ diffusion using a liquid $\mathrm{BBr}_{3}$ source in two different groups to form a symmetrical structure of $\mathrm{p}^{+} / \mathrm{n} / \mathrm{p}^{+}$for lifetime measurement. The emitter profiles were determined using spreading resistance and are shown in Figure 2. The surface concentrations obtained after deglaze for groups A and B are approximately $5 \times 10^{19} \mathrm{~cm}^{-3}$ and $6 \times 10^{18} \mathrm{~cm}^{-3}$ with corresponding sheet resistances $R_{s h}$ of $200 \Omega / \square$ and $150 \Omega / \square$, respectively. All samples received a clean HF dip prior to nitride depositions.

All samples received subsequent silicon nitride depositions on both sides, one side at a time. The film deposition was performed in Roth \& Rau high-frequency microwave remote PECVD systems. For most samples, the deposition process was carried out at a temperature of $450^{\circ} \mathrm{C}$, a processing pressure of $0.2 \mathrm{mbar}$, a microwave frequency of $2.45 \mathrm{GHz}$, a total microwave power of $1000 \mathrm{~W}$, and $\mathrm{SiH}_{4}$ to $\mathrm{NH}_{3}$ gas mixture ratio of $1: 1$. This process gave $\sim 70 \mathrm{~nm}$ thick films with a refractive index of nearly 2 . For some samples, a slightly different set of deposition parameters was used, with a deposition temperature of $400^{\circ} \mathrm{C}$, a microwave power of $750 \mathrm{~W}$ and a slightly lower $\mathrm{SiH}_{4}$ to $\mathrm{NH}_{3}$ ratio, resulting in $\sim 90 \mathrm{~nm}$ thick films with an almost identical refractive index. 


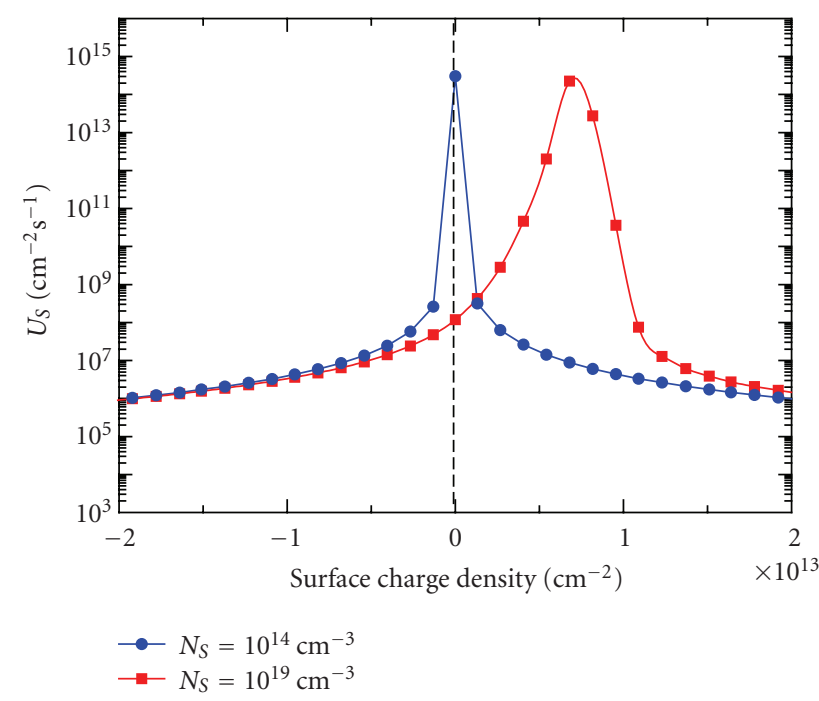

FIgure 1: Calculated dependence of surface recombination rate as a function of applied surface charge upon p-type surface with different surface doping. This calculation was performed using the extended Girisch model $[5,6]$ with a bulk injection level $10^{12} \mathrm{~cm}^{-3}$ and assuming a single level defect at midgap with equal capture cross sections.

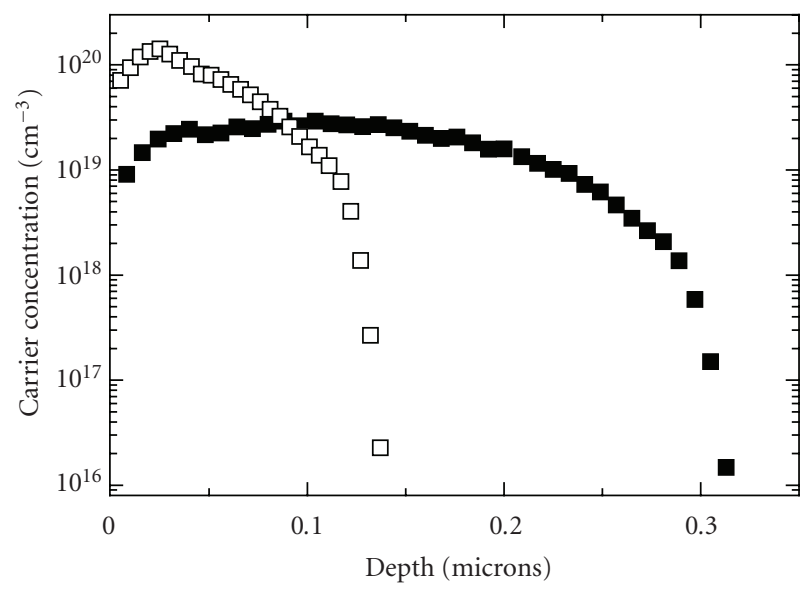

$\square$ Sample A
- Sample B

Figure 2: Boron emitter profiles of the samples used in this paper as determined by spreading resistance analysis.

The samples were characterized by effective lifetime measurements determined from photoconductance measurements in high level injection. The emitter saturation current density was extracted from the slope of the Auger corrected inverse lifetime as a function of injection level [13]. This was followed by surface charge deposition using a corona discharge chamber. The variation in the density of the deposited charge was $\pm 20 \%$. Kelvin probe measurements were performed immediately following charge deposition to monitor the surface potential change and thus allow calculation of deposited charge density at the surface. The surface charge density was subsequently calculated from the measured surface potential change, using appropriate values for the dielectric constants $\varepsilon_{\mathrm{SiN}_{x}}$ determined separately from capacitance-voltage measurements.

Czochralski (Cz), n-type, (100), 2-9 ohm $\cdot \mathrm{cm}, \sim 500 \mathrm{um}$ thick $c$-Si wafers were used for Capacitance-Voltage (C$\mathrm{V})$ measurements. Theses samples received a standard saw damage etch, RCA clean, and clean HF dip prior to PECVD nitride deposition. Nitride films with thickness around $100 \mathrm{~nm}$ were subsequently deposited on one polished side of the wafer, followed by $\mathrm{Al}$ metal dots (with diameter $700 \mathrm{um}$ ) evaporated on top of the surface to form a MetalInsulator-Semiconductor structure. Gallium-indium paste was applied on the rear side to allow electrical contact to Si bulk. Quasistatic (QS) and high-frequency (HF) C-V measurements were carried out.

\section{Results and Discussion}

4.1. Charge Density. Figure 3 shows typical quasi-static and high frequency $\mathrm{C}-\mathrm{V}$ curves of an as-deposited nitride film. The curves shown were measured with two different sweep directions. In addition to some hysteresis which is commonly observed, the shape of the curves shown is significantly different for the two sweep directions. A possible explanation for this unusual behavior could be the presence of interface defects with relatively small capture cross sections, and additionally with $\sigma_{p} \ll \sigma_{n}$. As the Fermi level is swept from near the valence band to the conduction band edge, filling of the traps with electrons is sufficiently rapid to result in high QS capacitances and a stretch-out of the HF curve. However, as the Fermi level is swept in the other direction, emission from the traps is much slower, resulting in the modified shape of the curve. These traps are mostly removed following an anneal in $\mathrm{N}_{2}$ at $400^{\circ} \mathrm{C}$, resulting in the curves shown in Figure 4.

The values of the accumulation capacitance (equal to the insulator capacitance $C_{i}$ ) and the flat band voltage $V_{\mathrm{fb}}$ allow determination of the charge density $Q_{f}$ in the as-deposited and annealed films, using:

$$
\begin{aligned}
\Delta \varphi_{m s} & =\varphi_{m}-\left[\chi_{\mathrm{Si}}+\frac{\left(E_{c}-E_{f}\right)}{q}\right], \\
Q_{f} & =\left(\Delta \varphi_{m s}-V_{\mathrm{fb}}\right) \frac{C_{i}}{A q},
\end{aligned}
$$

where $A$ is the capacitor area $\left(0.0039 \mathrm{~cm}^{2}\right), \varphi_{m s}$ is the Alsilicon work function difference, $\varphi_{m}$ is the work function of $\mathrm{Al}(4.1 \mathrm{eV}), \chi_{\mathrm{Si}}$ is the electron affinity of $\mathrm{Si}(4.05 \mathrm{eV}), q$ is the electronic charge, and $E_{c}-E_{f}$ is the difference between the conduction band edge and fermi level in the material used $(0.24 \mathrm{eV})$. Given the flat band voltage $\left(V_{\mathrm{fb}}\right)$ obtained from $\mathrm{C}-\mathrm{V}$ data, the fixed charge density within the nitride films used in this paper was calculated using (3). The results are summarized in Table 1. As can be seen, both the as-deposited and annealed films display a relatively low-charge density.

4.2. The Effect of a Boron Diffused Surface. Figure 5 shows curves of Auger corrected inverse lifetime as a function of 


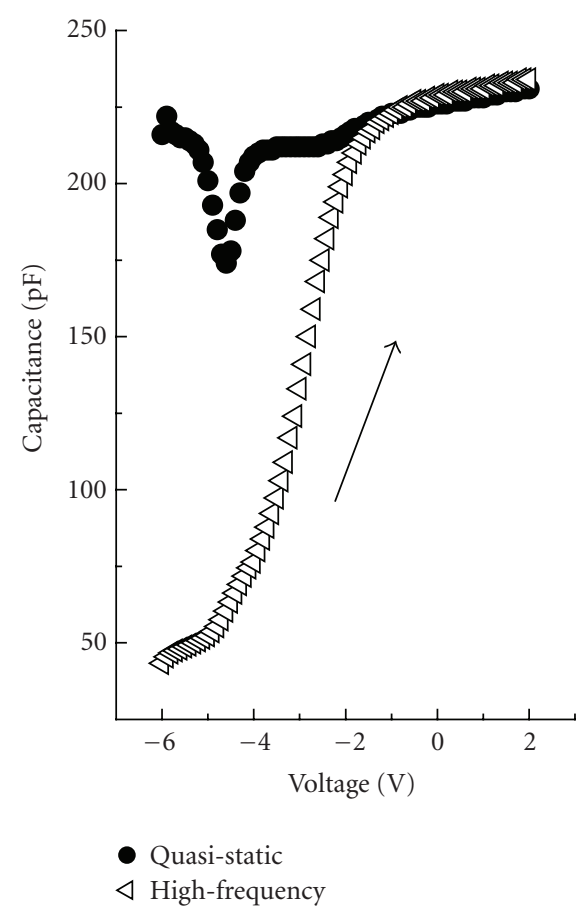

(a)

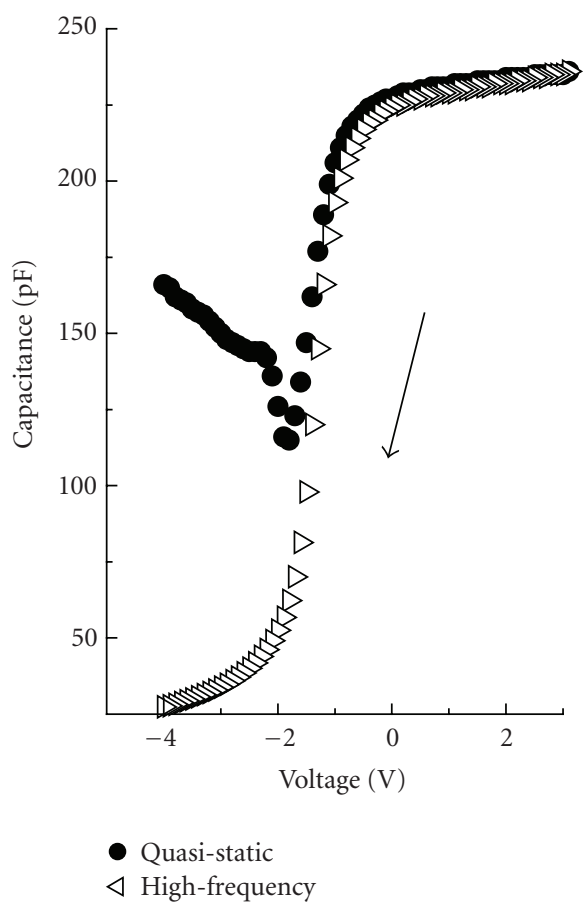

(b)

FIgure 3: Quasi-static and high-frequency C-V curves of a PECVD nitride film deposited at $450^{\circ} \mathrm{C}$. The voltage during the measurements was swept: (a) from inversion to accumulation, and (b) from accumulation to inversion.

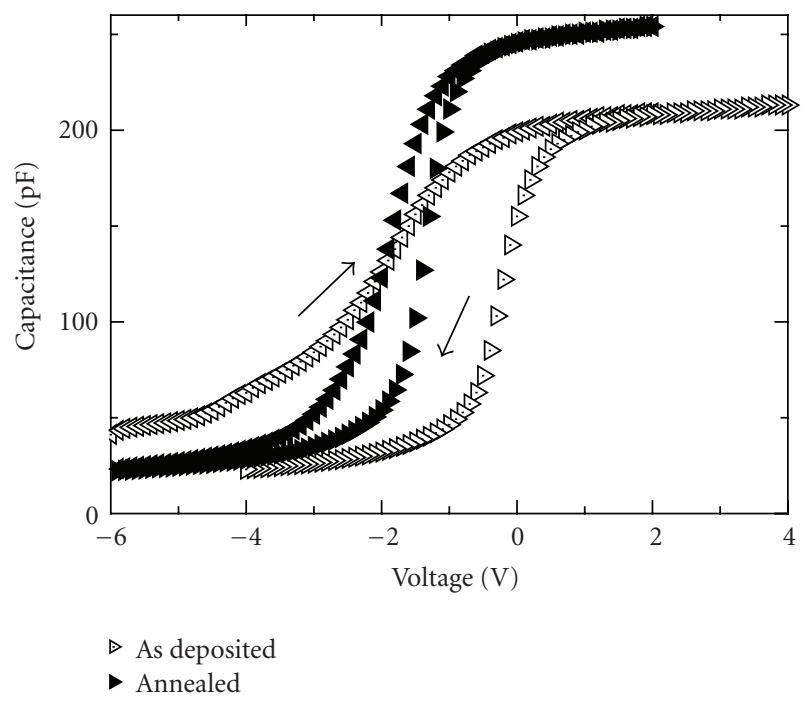

Figure 4: High-frequency C-V curves measured for different sweep directions on as-deposited (open symbols) and annealed (filled symbols) nitride films deposited at $450^{\circ} \mathrm{C}$.

excess carrier density for a 40-60 $\Omega \cdot \mathrm{cm}$, planar, n-type undiffused (100) sample following PECVD nitride deposition, as well as after subsequent corona charging. Following the deposition of high negative or positive charge densities, the surface is expected to be in low level injection. The net charge density under these conditions is estimated to be $-4.7 \times$ $10^{12}$ and $+5.9 \times 10^{12} \mathrm{~cm}^{-2}$. These net charge densities were
TABLE 1: Flat band voltage and fixed charge density of the PECVD silicon nitride films used in this study as determined from $\mathrm{C}-\mathrm{V}$ measurements.

\begin{tabular}{lcccc}
\hline Deposition & \multicolumn{2}{c}{ As Deposited } & \multicolumn{2}{c}{ Annealed } \\
Temperature & $V_{\mathrm{fb}}(\mathrm{V})$ & $Q_{f}\left(\mathrm{~cm}^{-2}\right)$ & $V_{\mathrm{fb}}(\mathrm{V})$ & $Q_{f}\left(\mathrm{~cm}^{-2}\right)$ \\
\hline $450^{\circ} \mathrm{C}$ & -1.92 & $+6.5 \times 10^{11}$ & -1.54 & $+5.8 \times 10^{11}$ \\
$400^{\circ} \mathrm{C}$ & -1.22 & $+2 \times 10^{11}$ & -1.9 & $+4.3 \times 10^{11}$ \\
\hline
\end{tabular}

obtained from the sum of $Q_{f}$ (from $\mathrm{C}-\mathrm{V}$ measurements) and the applied surface charge density $Q_{s}$, obtained from the change in surface potential following corona charging as measured using a Kelvin probe. However, the actual value of net charge density may be overestimated by this method, particularly for large $Q_{s}$. This is because the application of large charge densities on the surface of the nitride can induce the injection of the opposite type of carrier into the nitride film. As a result of such injection, it is observed in Figure 5 that the removal of negative surface charge by rinsing in isopropyl alcohol (IPA) results in a lifetime curve which approaches that following positive corona charging (due to the injection and storage of positive charge in the nitride) while the removal of positive surface charge results in a lifetime curve which approaches that following negative corona charging (due to the injection and storage of negative charge in the nitride).

The emitter saturation current density $\left(J_{0 e}\right)$ can be determined from the slope of the Auger-corrected inverse lifetime curves. As shown in Figure 5, both after negative 


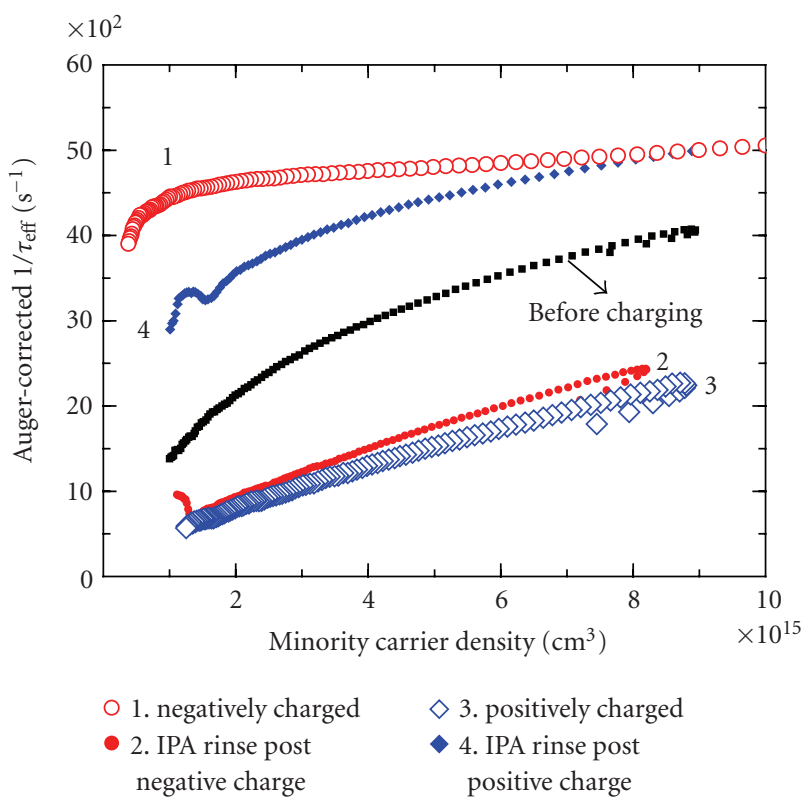

FIGURE 5: Plots of Auger corrected-inverse lifetime as a function of injection level measured on a PECVD $\mathrm{SiN}_{x}$ passivated n-type, (100) oriented Si sample. The films were deposited at $400^{\circ} \mathrm{C}$. The inserted numbers represent the sequence of the charging steps. Blackstraight line represents the initial measurement which is followed by consecutive negative charge deposition (red-circle), rinse in IPA (black-circles), positive charge deposition (blue-squares), and another rinse in IPA (black squares).

and positive charging, the Auger corrected inverse lifetime plots yield reasonably straight lines at high injection levels, as would be expected for a sample in which the surface is in low-level injection while the bulk is in high-level injection. Nevertheless, following negative corona charging, an unusual feature was observed. While $J_{0 e}$ extracted from the slope of inverse lifetime is very low, (in fact, $J_{0 e}$ following negative corona charge application is significantly lower than following positive charge application) the effective lifetime is significantly lower as well. This unusual behaviour cannot be explained by recombination in the depletion region created by the surface inversion, since, under conditions of high level injection, no depletion region exists in the samples.

In order to analyse the results from minority carrier lifetime measurements, two methods were used to extract $S_{\text {eff }}$ over the range of applied charge densities of the same sample as in Figure 5. The first is to determine $S_{\text {eff }}$ from the measurement of $J_{0 e}$ :

$$
S_{\text {eff }}=\frac{J_{0 e}\left(N_{A}+\Delta n\right)}{q n_{i}^{2}}
$$

where $\Delta n$ is the excess carrier density in the sample bulk and $N_{A}$ is the background doping concentration. $S_{\text {eff }}$ was determined at an injection level $4 \times 10^{15} \mathrm{~cm}^{-3}$ and thus, $N_{A}$ is small compared to $\Delta n$. The determination of $S_{\text {eff }}$ from $J_{0 e}$ is not expected to be accurate over the entire range of applied charge densities. The condition for valid $J_{0 e}$ measurementsthat the surface must be in low level injection-will not be achieved for low-net charge densities, (less than about $\pm 1 \times$ $10^{12} \mathrm{~cm}^{-2}$ ). However, for large densities of either negative or positive charge, the presence of an accumulation or inversion layer should result in a meaningful extraction of $J_{0 e}$.

The second method is to use the relationship between bulk lifetime, effective (measured) lifetime, and surface recombination [14] which is given by:

$$
\frac{1}{\tau_{\mathrm{eff}}}=\frac{1}{\tau_{b}}+\beta^{2} D_{\mathrm{amb}}
$$

where $\beta$ is expressed by

$$
\tan \left(\frac{\beta W}{2}\right)=\frac{S_{\mathrm{eff}}}{\beta D_{\mathrm{amb}}} .
$$

By combining (5) and (6), $S_{\text {eff }}$ can be calculated by

$$
S_{\text {eff }}=\sqrt{D_{\mathrm{amb}}\left(\frac{1}{\tau_{\mathrm{eff}}}-\frac{1}{\tau_{b}}\right)} \tan \left[\frac{W}{2} \sqrt{\frac{1}{D_{\mathrm{amb}}}\left(\frac{1}{\tau_{\mathrm{eff}}}-\frac{1}{\tau_{b}}\right)}\right] .
$$

Here, $D_{\text {amb }}$ is the ambipolar diffusion constant $\left(\mathrm{cm}^{2} / \mathrm{s}\right), W$ is wafer thickness, and $\tau_{b}$ is the bulk lifetime. This equation assumed that all recombination can be attributed to either the bulk or the surface region, and that no other recombination source (e.g., depletion recombination) is present. Equation (7) requires determination of $\tau_{b}$. We estimate $\tau_{b}$ for each sample using (8) in which $S_{\text {eff }}$ is calculated with (4) using the highest $\tau_{\text {eff }}$ which was obtained under accumulation conditions, with positive applied charge.

$$
\frac{1}{\tau_{\text {eff }}}=\frac{1}{\tau_{b}}+\frac{2 S_{\text {eff }}}{W} .
$$

Equation (7) is used to calculate $S_{\text {eff }}$ as a function of $\tau_{\text {eff }}$, rather than simpler and more commonly used expression in (8). This is done since, for several of the samples analysed, the relatively high-surface recombination velocities lead to a significant $(>10 \%)$ underestimation of $S_{\text {eff }}$ if $(8)$ is used.

As shown in Figure 6, it is clear that the application of negative charge leads to significant discrepancy between the two calculations due to the opposing trends of decreasing $J_{0 e}$ and decreasing $\tau_{\text {eff }}$, which cannot be reconciled with a simple model of a sample with a uniform bulk and surfaces in low level injection. Similar results have been observed in a range of PECVD nitride passivated, undiffused samples deposited using different PECVD systems and on different substrates, including both lightly and moderately doped, $\mathrm{p}$ type and $\mathrm{n}$ type substrates. It is confined to undiffused substrates with a PECVD nitride layer, following the deposition of negative charge. The reason for this phenomenon is currently not well understood and will require further investigation. In this paper, we will not concern ourselves further with measurements on undiffused substrates following the application of negative charge. In the subsequent discussions, (6) will be used for $S_{\text {eff }}$ extraction, since this method does not require the surface to be in low level injection.

The variation of $S_{\text {eff }}$ with net charge density for a B diffused sample with a high surface $B$ concentration 


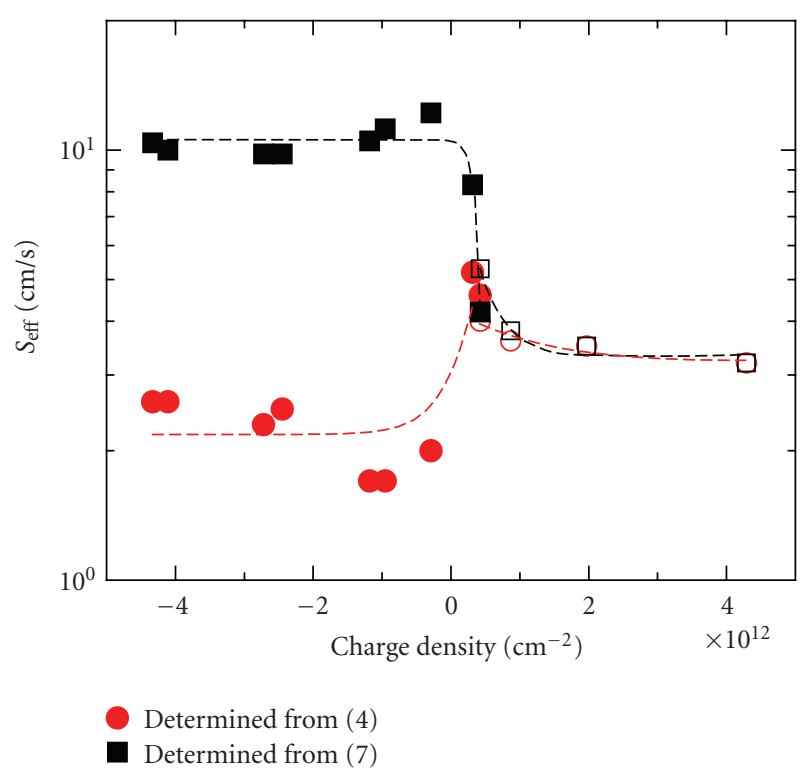

FIGURE 6: $S_{\text {eff }}$ as a function of applied charge density calculated from $J_{0 e}(4)$ and $\tau_{\text {eff }}(7)$ with respect to applied surface charge. PECVD $\mathrm{SiN}_{x}$ was deposited at $400^{\circ} \mathrm{C}$ and the sample was subsequently annealed ( 30 mins $400^{\circ} \mathrm{C}$ in $\mathrm{N}_{2}$ ). Negative and positive charge application is denoted by filled and empty symbols, respectively. All $S_{\text {eff }}$ values were extracted from data measured at an injection level $4 \times 10^{15} \mathrm{~cm}^{-3}$.

is depicted in Figure 7. Negative charges were initially deposited, followed by charge removal in IPA solution and deposition of positive charge (in according with the sequence numbers given on the curve). The starting point (point 1), prior to any charging, is given by $Q_{f}\left(+5.8 \times 10^{11} \mathrm{~cm}^{-2}\right)$. The results of Figure 6 show a reduction and eventual saturation of $S_{\text {eff }}$ as the surface is pushed into accumulation. This trend is consistent with other reports in the literature [8-12]. We applied the extended Girisch model $[5,6]$ to simulate the behaviour. Figure 7 shows that a reasonably good fit to the data can be obtained when a suitable set of modelling parameters is chosen. The distribution of electrons and holes capture cross sections and the density of dopantlike defects over the bandgap energy that were used in this model are given in Figure 8. However, it is not possible to make definitive statements concerning the variation of defect density or capture cross sections from the available data. As mentioned before, the Girisch model predicts a continuing decrease in $S_{\text {eff }}$ as the negative charge density is increased, while in practice, saturation of $S_{\text {eff }}$ is observed.

It is interesting to note from the experimental results shown in Figure 7 that, even for a very heavily doped surface, the application of charge can still result in a significant further reduction in surface recombination. Previous studies $[10,12]$ on undiffused surfaces have found that surface recombination tends to saturate for applied surface charge densities of $5 \times 10^{12} \mathrm{~cm}^{-2}$ or less, with corresponding surface majority carrier concentrations of $5 \times 10^{19} \mathrm{~cm}^{-3}$ or less. For the above sample, $S_{\text {eff }}$ is observed to saturate for charge densities of $\sim 5 \times 10^{12} \mathrm{~cm}^{2}$, but the corresponding surface majority carrier concentrations are significantly higher than

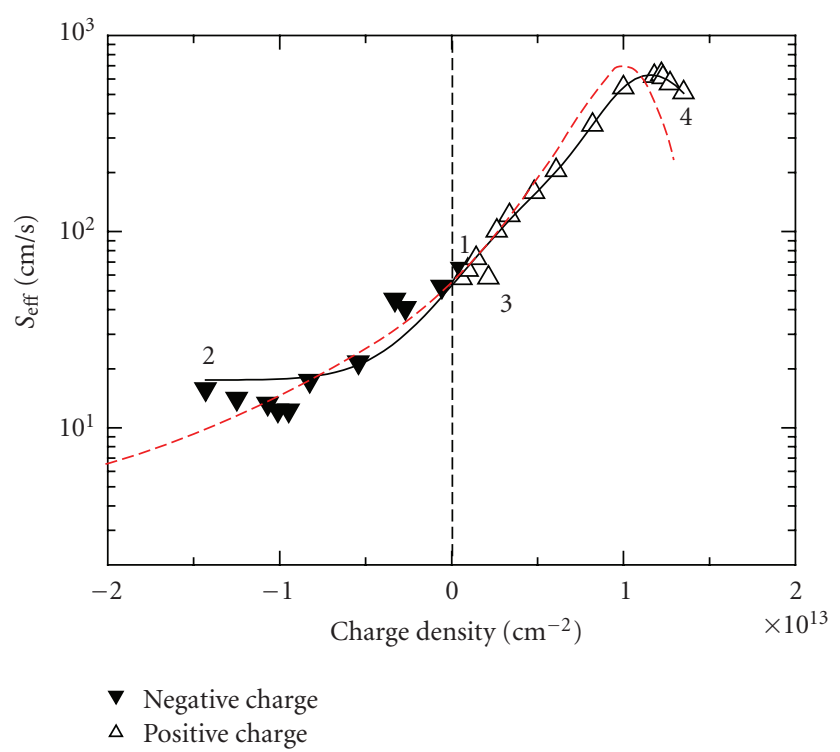

Figure 7: $S_{\text {eff }}$ as a function of applied charge density for a B diffused annealed sample with $N_{s} \approx 5 \times 10^{19} \mathrm{~cm}^{-3}$. PECVD SiN $x$ was deposited at $450^{\circ} \mathrm{C}$ and the sample was subsequently annealed ( 30 mins $400^{\circ} \mathrm{C}$ in $\mathrm{N}_{2}$ ). Negative and positive charge application is denoted by filled and empty symbols, respectively. The black solid line is a fit to the data, while the red-dashed line shows the modelling results obtained from the Girisch model $[5,6]$, assuming a continuum of defect levels with midgap ratio $\sigma_{n} / \sigma_{p} \approx 10^{2}$ and $D_{\text {itd }}$ $=2 \times 10^{10} \mathrm{~cm}^{-2} \mathrm{eV}^{-1}$. This density and capture cross sections of the defects were adjusted to fit the experimental data.

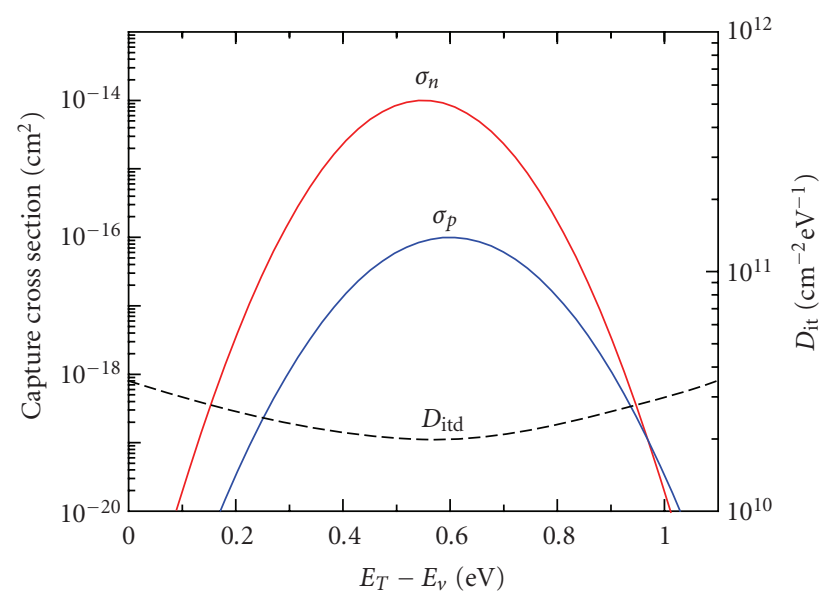

Figure 8: Modelled energy dependence of the capture cross sections of electrons $\left(\sigma_{n}\right)$ and holes $\left(\sigma_{p}\right)$ and dopant-like defect density $\left(D_{\text {itd }}\right)$ that were used to fit the experimental data as given in Figure 7.

$5 \times 10^{19} \mathrm{~cm}^{-3}$. The main conclusion that can be drawn from Figure 7 is that even heavily doped surfaces are sensitive to the presence of charge.

The best $J_{0 e}$ of the B diffused sample shown in Figure 7 following negative charge application is approximately $41 \mathrm{fA} / \mathrm{cm}^{2}$. This can be compared to a $J_{0 e}$ of $\sim 6 \mathrm{fA} / \mathrm{cm}^{2}$ for an identically prepared, undiffused sample under accumulation. The difference in $J_{0 e}$ values is partly due to the contribution 


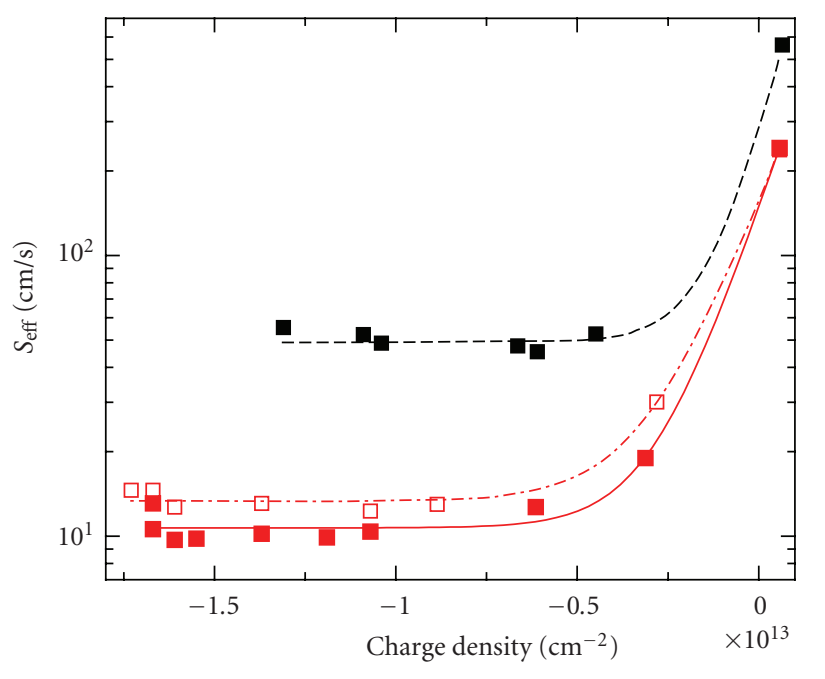

- As deposited

- Annealed in $\mathrm{N}_{2}$

口 Annealed in FGA

FIGURE 9: $S_{\text {eff }}$ as a function of charge density for a B diffused sample with $N_{s} \approx 6 \times 10^{18} \mathrm{~cm}^{-3}$ and PECVD SiN $x$ was deposited at $450^{\circ} \mathrm{C}$. The measurements were taken at injection level $4 \times 10^{15} \mathrm{~cm}^{-3}$ in asdeposited condition and after annealing in different ambient. The lines are fit to the data.

to $J_{0 e}$ from the emitter region so this factor should be accounted. Results on oxide passivated B diffused emitters with similar sheet resistances have shown that this kind of emitter contributes less than $6 \mathrm{fA} / \mathrm{cm}^{2}$ [15]. The remainder of the difference shown in this experiment $\left(\sim 29 \mathrm{fA} / \mathrm{cm}^{2}\right)$ is likely to indicate that the B diffused interface is not as good as that of undiffused (i.e., has a higher interface defect density, or defects with greater capture cross sections), as has also been observed for samples passivated with a thermal oxide [16].

Figure 9 shows the $S_{\text {eff }}$ extractions for a B diffused sample with nitride film deposited at $450^{\circ} \mathrm{C}$ before and after anneals at a temperature of $400^{\circ} \mathrm{C}$ for 30 minutes. These anneals were performed in $\mathrm{N}_{2}$. This was followed by an annealing in FGA on the same sample. Annealing in $\mathrm{N}_{2}$ was able to improve the interface quality of both diffused and undiffused surfaces, as indicated by lower $S_{\text {eff }}$ regardless of the charge density on the surface. This improvement is in good agreement with the results shown in Reference [2]. Furthermore, it is also shown by the results given in Figure 9 that the introduction of $\mathrm{H}$ molecules did not contribute to the passivation, since there is no further improvement shown following second annealing in forming gas. Therefore, it is suggested that the improved interface quality is more likely due to rearrangements and the removal of some defects at the interface at elevated temperature, as well as possibly the passivation of interface defects with hydrogen supplied by the $\mathrm{SiN}_{x}$ film.

4.3. The Effect of Surface Doping and Surface Orientation. The effect of B surface doping level on different surface

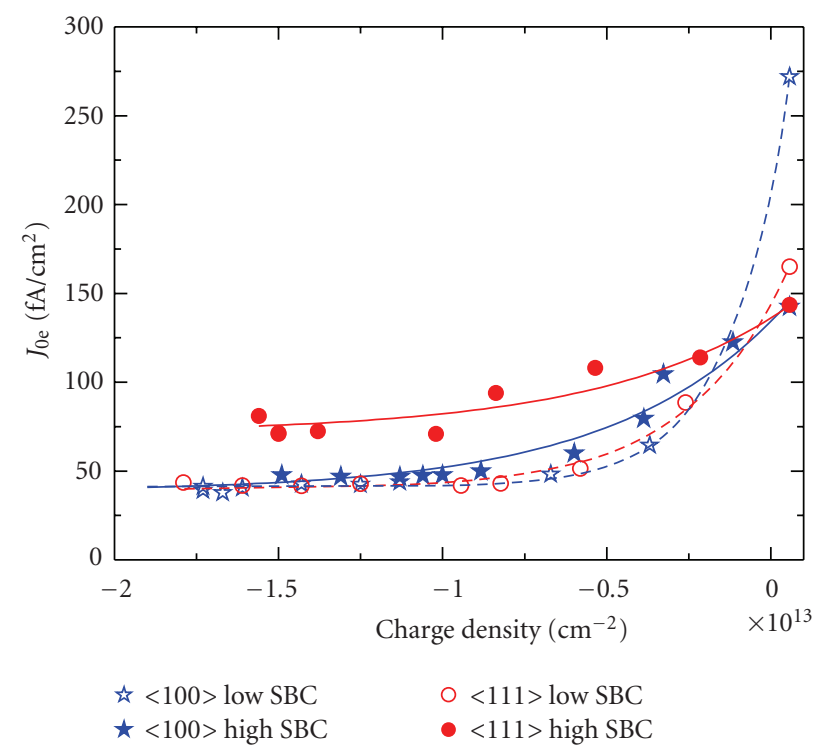

FIGURE 10: $J_{0 e}$ as a function of charge density of (100) and (111) B diffused planar samples with different surface boron concentration (SBC). "Low" and "high" SBC refers to $N_{s}=6 \times 10^{18}$ and $5 \times$ $10^{19} \mathrm{~cm}^{-3}$ on (100) samples, respectively. All samples were annealed in $\mathrm{N}_{2}$ prior to charge deposition. Measurements were taken at an injection level $4 \times 10^{15} \mathrm{~cm}^{-3}$.

orientations as a function of net charge density is plotted in Figure 10. These samples received anneals in $\mathrm{N}_{2}$ at $400^{\circ} \mathrm{C}$.

At low charge density where $Q_{s}=0, J_{0 e}$ for both (100) and (111) samples is dependent on surface doping concentration, with a lower B surface concentration resulting in a higher $J_{0 e}$ due to a lower hole concentration (and hence a higher minority carrier electron concentration), which results in higher recombination. For the case of (100), the $J_{0 e}$ values for a high applied density of negative charge are almost identical; suggesting that the difference in B surface concentration has not significantly affected the interface defect properties, such as their density, distribution, or capture cross sections. Given that the presence of a B diffusion clearly degrades the interface, it may be that the extent of this degradation is more closely related to the total $\mathrm{B}$ concentration rather than $\mathrm{B}$ concentration at the surface. However, further work will be required to verify this.

For the (111) samples, the dependence of $J_{0 e}$ on surface concentration under no charge application is much less pronounced. In contrast to the (100) surface, the final accumulated $J_{0 e}$ values of (111) samples are found to be dependent on surface concentration, with a higher surface concentration resulting in higher saturated $J_{0 e}$ values. This result suggests that the interface properties of the (111) surface are more susceptible to B surface concentration than the (100) surface. For low B surface concentrations, there appears to be no significant difference in the passivation quality of (100) and (111) surfaces, suggesting that, for lowly and moderately doped surfaces, the PECVD $\operatorname{SiN}_{x}$ passivation is rather independent of surface orientation. 


\section{Conclusion}

In conclusion, we have demonstrated that the application of positive surface charge results in a significant increase in the surface recombination of PECVD $\mathrm{SiN}_{x}$ passivated $\mathrm{B}$ diffused emitters, even for high $B$ surface concentrations. On the other hand, negative charge causes a substantial reduction in surface recombination. Under accumulation, the significant difference in $J_{0 e}$ between $\mathrm{B}$ diffused and undiffused sample, as much as $\sim 29 \mathrm{fA} / \mathrm{cm}^{2}$, implies that the $\mathrm{B}$ diffusion has resulted in some degradation of the Si-PECVD $\mathrm{SiN}_{x}$ interface. The very similar passivation quality of (100) samples with different B concentrations at the surface suggests that the degradation is not strongly related to the $\mathrm{B}$ surface concentration. However, the interface properties of (111) surfaces tend to be more susceptible to the $\mathrm{B}$ concentration at the surface rather than (100).

There are several possible implications for solar cell design. If PECVD $\operatorname{SiN}_{x}$ films were to be applied to the passivation of $\mathrm{B}$ diffused emitters, then minimising the positive charge density in the $\mathrm{SiN}_{x}$ film will be very important in order to obtain good surface passivation, even for a high B surface concentration. The results suggest that the B surface concentration should be rather high, even though-for some surfaces at least - a higher B concentration will lead to a poorer interface. It is likely that textures that avoid the formation of (111) surfaces will perform better than the random pyramid texture.

\section{Acknowledgments}

The authors wish to thank members of the PV group at the University of New South Wales for carrying out some of the PECVD depositions; Dr. Jason Tan and Mr. Chun Zhang for help and discussion on PECVD nitride depositions; and Mr. Simeon Baker-Finch and Dr. Keith McIntosh for discussions on emitter recombination. Financial support by the Australian Agency for International Development is gratefully acknowledged.

\section{References}

[1] J. Schmidt, K. Bothe, R. Bock, C. Schmiga, R. Krain, and R. Brendel, "N-type silicon-the better material choice for industrial high efficiency solar cells," in Proceedings of the 22nd European Photovoltaic Solar Energy Conference and Exhibition, Milan, Italy, September 2007.

[2] F. W. Chen, T.-T. A. Li, and J. E. Cotter, "Passivation of boron emitters on n-type silicon by plasma-enhanced chemical vapor deposited silicon nitride," Applied Physics Letters, vol. 88, no. 26, Article ID 263514, 2006.

[3] W. D. Eades and R. M. Swanson, "Calculation of surface generation and recombination velocities at the $\mathrm{Si}-\mathrm{SiO}_{2}$ interface," Journal of Applied Physics, vol. 58, no. 11, pp. 4267-4276, 1985.

[4] E. Yablonovitch, R. M. Swanson, W. D. Eades, and B. R. Weinberger, "Electron-hole recombination at the $\mathrm{Si}-\mathrm{SiO}_{2}$ interface," Applied Physics Letters, vol. 48, no. 3, pp. 245-247, 1986.

[5] R. B. M. Girisch, R. P. Mertens, and R. F. De Keersmaecker, "Determination of $\mathrm{Si}_{-} \mathrm{SiO}_{2}$ interface recombination param- eters using a gate-controlled point-junction diode under illumination," IEEE Transactions on Electron Devices, vol. 35, no. 2, pp. 203-222, 1988.

[6] A. G. Aberle, S. Glunz, and W. Warta, "Impact of illumination level and oxide parameters on Shockley-Read-Hall recombination at the $\mathrm{Si}_{-} \mathrm{SiO}_{2}$ interface," Journal of Applied Physics, vol. 71, no. 9, pp. 4422-4431, 1992.

[7] D. K. Schroder, "Contactless surface charge semiconductor characterization," Materials Science and Engineering B, vol. 91, pp. 196-210, 2002.

[8] M. Schoefthaler, R. Brendel, G. Langguth, and J. H. Werner, "High-quality surface passivation by corona-charged oxides for semiconductor surface characterization," in Proceedings of the 1st World Conference on Photovoltaic Energy Conversion, vol. 2, pp. 1509-1512, Waikoloa, Hawaii, USA, 1994.

[9] S. W. Glunz, D. Biro, S. Rein, and W. Warta, "Field-effect passivation of the $\mathrm{SiO}_{2}$-Si interface," Journal of Applied Physics, vol. 86, no. 1, pp. 683-691, 1999.

[10] W. E. Jellett and K. J. Weber, "Accurate measurement of extremely low surface recombination velocities on charged, oxidized silicon surfaces using a simple metal-oxidesemiconductor structure," Applied Physics Letters, vol. 90, no. 4, article 042104, 3 pages, 2007.

[11] S. Dauwe, J. Schmidt, A. Metz, and R. Hezel, "Fixed charge density in silicon nitride films on crystalline silicon surfaces under illumination," in Proceedings of the 29th IEEE Photovoltaic Specialists Conference, pp. 162-165, New Orleans, La, USA, 2002.

[12] K. J. Weber, H. Jin, C. Zhang, N. Nursam, W.E. Jellett, and K. R. McIntosh, "Surface passivation using dielectric films: how much charge is enough," in Proceedings of the 24th European Photovoltaic Solar Energy Conference and Exhibition, Hamburg, Germany, 2002.

[13] D. E. Kane and R. M. Swanson, "Measurement of the emitter saturation current by a contactless photoconductivity decay method," in Proceedings of the 18th IEEE Photovoltaic Specialists Conference, pp. 578-583, Las Vegas, Nev, USA.

[14] A. W. Stephens, A. G. Aberle, and M. A. Green, "Surface recombination velocity measurements at the silicon-silicon dioxide interface by microwave-detected photoconductance decay," Journal of Applied Physics, vol. 76, no. 1, pp. 363-370, 1994.

[15] W. E. Jellet, Investigation of recombination at the siliconsilicon dioxide interface, Ph.D. thesis, The Australian National University, Canberra, Australia, 2008.

[16] W. E. Jellett, K. J. Weber, and H. Jin, "Influence of boron diffusion on the Si surface passivation," in Proceedings of the 22nd European Photovoltaic Solar Energy Conference and Exhibition, Milan, Italy, 2007. 

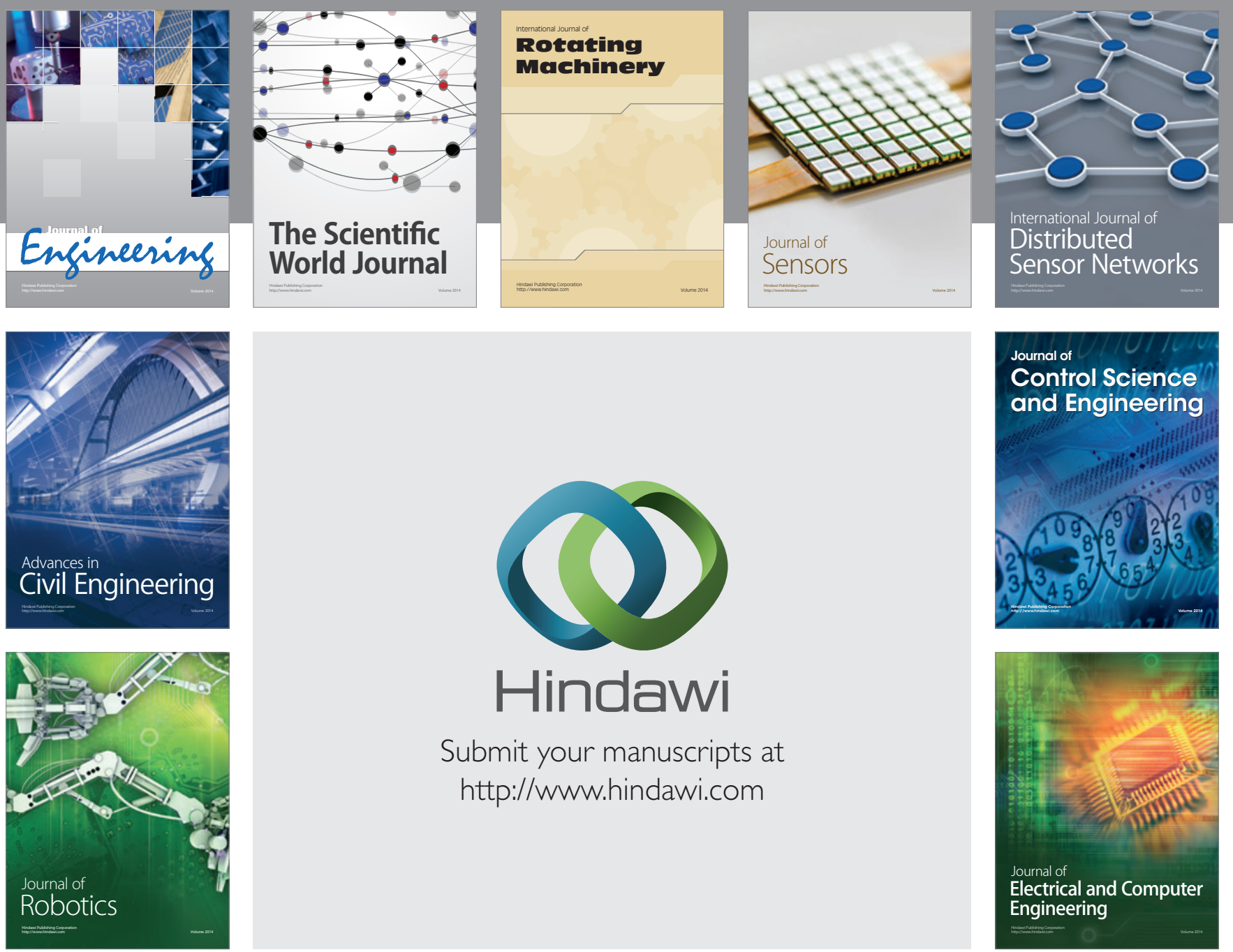

Submit your manuscripts at

http://www.hindawi.com
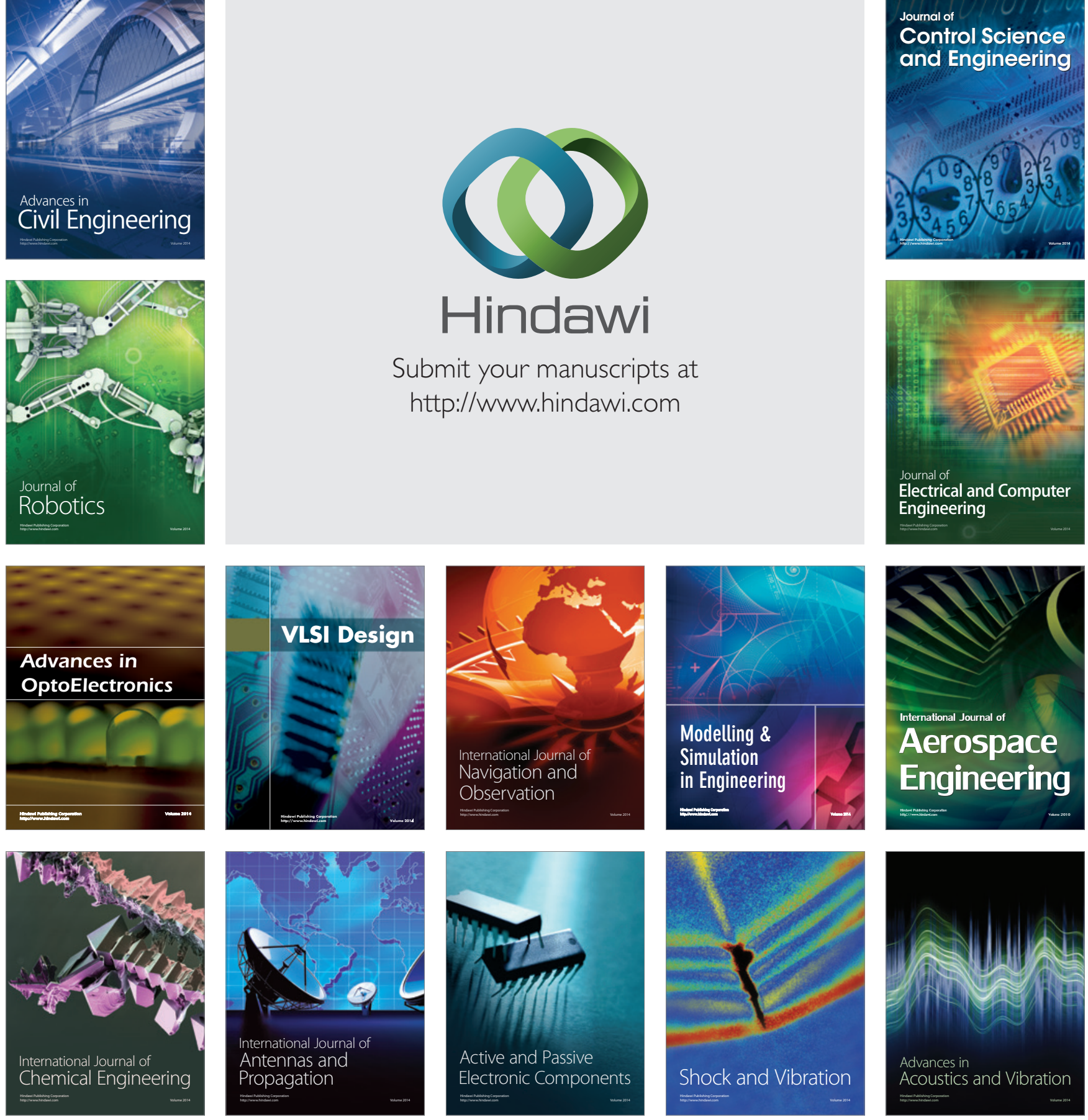